\title{
Critical Temperature and Enhanced Isotope Effect in the Presence of Paramagnons in Phonon-Mediated Superconductors
}

\author{
O. V. Dolgov, ${ }^{1}$ I. I. Mazin, ${ }^{2}$ A. A. Golubov, ${ }^{3}$ S. Y. Savrasov, ${ }^{4}$ and E. G. Maksimov ${ }^{5}$ \\ ${ }^{1}$ Max-Planck-Institut für Festkörperphysik, Heisenbergstrasse 1, 70569 Stuttgart, Germany \\ ${ }^{2}$ Center for Computational Materials Science, Naval Research Laboratory, Washington, D.C. 20375, USA \\ ${ }^{3}$ Faculty of Science and Technology, University of Twente, 7500 AE Enschede, The Netherlands \\ ${ }^{4}$ Department of Physics, University of California, Davis, California 95616, USA \\ ${ }^{5}$ P. N. Lebedev Physical Institute, RAS, Leninskii prospekt 53, 119991 Moscow, Russia
}

(Received 14 June 2005; published 15 December 2005)

\begin{abstract}
We reconsider the long-standing problem of the effect of spin fluctuations on the critical temperature and isotope effect in a phonon-mediated superconductor. Although the general physics of the interplay between phonons and paramagnons has been rather well understood, the existing approximate formulas fail to describe the correct behavior of $T_{c}$ for general phonon and paramagnon spectra. Using a controllable approximation, we derive an analytical formula for $T_{c}$ which agrees well with exact numerical solutions of the Eliashberg equations for a broad range of parameters. Based on both numerical and analytical results, we predict a strong enhancement of the isotope effect when the frequencies of spin fluctuation and phonons are of the same order. This effect may have important consequences for nearmagnetic superconductors such as $\mathrm{MgCNi}_{3}$.
\end{abstract}

DOI: 10.1103/PhysRevLett.95.257003

In the last decade, a large number of superconductors were discovered in which enhanced spin fluctuations (SF) play a role in the superconductivity, e.g., $\mathrm{Sr}_{2} \mathrm{RuO}_{4}$, $\mathrm{MgCNi}_{3}, \varepsilon-\mathrm{Fe}, \mathrm{ZrZn}_{2}$, and others, bringing about new and interesting physics. However, understanding such materials, even at an intuitive level, has been hindered by the lack of a simple formula that would approximate the full Eliashberg theory in a compact analytical form, as the conventional McMillan formula (MMF) does. As a result, uncritical generalizations of the latter have been used as a substitute, despite the fact that, as we will show below, some of them are too approximate or outright incorrect. In this Letter we present an analogue of the MMF, derived in a controllable way and tested against numerical solutions of full Eliashberg equations, including interaction with SF (paramagnons). We point out the possibility of a giant phonon isotope effect induced by $S F$. We will also apply this theory, as an example, to a nearly ferromagnetic superconductor, $\mathrm{MgCNi}_{3}$.

The understanding that SF are pair breakers in conventional superconductors is nearly as old as the BCS theory itself [1]. Moreover, it was soon realized that strong coupling manifests itself in a nontrivial way in the presence of SF [2,3]. In a number of papers, numerical solutions of the Eliashberg equations were presented, incorporating phonon $\alpha^{2} F_{p}(\omega)$ as well as $\mathrm{SF} \alpha^{2} F_{s}(\omega)$ spectral functions (see, e.g., Ref. [4]). However, solving the full Eliashberg equations is not always an option and does not provide as much physical insight as an analytical treatment. An analytical tool comparable to the famed MMF is needed.

Retrospectively, one can realize that the overwhelming success of the MMF is due to three facts: (a) it can be derived analytically using simple approximations; (b) it includes Coulomb repulsion effects; (c) it has three uni-
PACS numbers: 74.20.Mn, 74.62.-c, 74.70.-b

versal adjustable parameters, which, after little tuning, produce an expression which is surprisingly accurate for a large variety of spectra. Compared to the BCS equation, the MMF includes three essential pieces of additional physics: effective mass renormalization, reduction of the Coulomb repulsion, and proper (logarithmic) averaging of the phonon frequency. All three effects can be derived analytically in some approximations. In fact, the functional form of the MMF can be derived in two different ways. One, known as the square-well model [5], uses the Matsubara representation, where the coupling is parametrized in terms of the matrix $\lambda\left(n, n^{\prime}\right)$. The model assumes two different approximations for the same function $\lambda\left(n, n^{\prime}\right)$, depending on whether it is used in the equation for the mass renormalization $Z$ or in the one for the gap function $\phi$ :

$$
\begin{aligned}
& \lambda_{Z}\left(n, n^{\prime}\right)=\lambda_{p} \Theta\left(\omega_{p}-\left|\omega_{n-n^{\prime}}\right|\right), \\
& \lambda_{\phi}\left(n, n^{\prime}\right)=\lambda_{p} \Theta\left(\omega_{p}-\left|\omega_{n}\right|\right) \Theta\left(\omega_{p}-\left|\omega_{n^{\prime}}\right|\right),
\end{aligned}
$$

leading to an equation for the critical temperature:

$$
T_{c}=a \omega_{\log } \exp \left\{-b\left(1+\lambda_{Z}\right) /\left[\lambda_{\phi}-\mu^{*}\left(1+c \lambda_{Z}\right)\right]\right\},
$$

where the theoretical parameters are $a=1.14, b=c=1$, $\lambda_{Z}=\lambda_{\phi}=\lambda_{p}=2 \int_{0}^{\infty} \omega^{-1} \alpha^{2} F_{p}(\omega) d \omega$, and $\lambda_{p} \ln \omega_{\log }=$ $2 \int_{0}^{\infty} \omega^{-1} \ln \omega \alpha^{2} F_{p}(\omega) d \omega$. The Coulomb potential is reduced from its bare value $\mu$ as $\mu^{*}=\mu /\left(1+\mu \ln \frac{\omega_{C}}{\omega_{\log }}\right)$, where $\omega_{C}$ is the frequency cutoff of the Coulomb interaction. The MMF formula is given by Eq. (2) with optimized parameters $a=1 / 1.2, b=1.04$, and $c=0.62$.

$\mathrm{SF}$, as opposed to phonons, induce repulsion for singlet pairs. However, they contribute to the mass renormalization just the same. Therefore the first instinct is to let $\lambda_{Z}=\lambda_{p}+\lambda_{s}$, where $\lambda_{s}$ describes the SF, and 
$\lambda_{\phi}=\lambda_{p}-\lambda_{s}$. Equation (2) with this modification and standard $a, b$, and $c$ is the one routinely used in the literature for materials with SF (e.g., Refs. [6-8]).

Obviously, using two different approximations for the same physical function $\lambda\left(n, n^{\prime}\right)$ depending on whether it appears in the first or second Eliashberg equation cannot be justified by any logic. It appears that the MMF formula can be fortuitously derived in this way, but, as we will see below, this approach fails when SF are included. An alternative derivation of the MMF utilizes the real frequency axis formalism [9]. One assumes an Einstein phonon at frequency $\omega_{p}$. The Eliashberg equations are then solved iteratively. After the first iteration, one obtains [9]

$$
T_{c}=1.14 \omega_{p} \exp \left\{-\frac{1}{2}-\frac{1+\lambda_{p}}{\lambda_{p}-\mu^{*}\left[1+0.5 \frac{\lambda_{p}}{1+\lambda_{p}}\right]}\right\},
$$

which is similar to the square-well formula Eq. (2) with $a=1.14 / \sqrt{e}=1 / 1.44$ (note that this value of $a$ is much closer to the optimized one), $b=1$, and $c=0.5 /\left(1+\lambda_{p}\right)$. This approach is a controllable approximation with a concrete physical meaning. However, it has never been applied to superconductors with SF.

On the contrary, several attempts to apply the squarewell model to SF have been reported. In Refs. $[3,4]$ the following expression was derived (for $\mu^{*}=0$ ):

$$
T_{c}=1.14 \omega_{p}^{\nu} \omega_{s}^{1-\nu} \exp \left(-\frac{1+\lambda_{p}+\lambda_{s}}{\lambda_{p}-\lambda_{s}}\right),
$$

where Carbotte et al. [4] suggested the following $\nu$ :

$$
\nu=\lambda_{p} /\left(\lambda_{p}-\lambda_{s}\right) .
$$

Vonsovsky et al. [3] proposed another expression:

$$
\nu=\frac{\lambda_{p}^{2}}{\lambda_{p}-\lambda_{s}}\left[\lambda_{p}-\lambda_{s}+\frac{\lambda_{p} \lambda_{s}}{1+\lambda_{p}+\lambda_{s}} \ln \frac{\omega_{p}}{\omega_{s}}\right]^{-1} .
$$

Unfortunately, neither Ref. [3] nor Ref. [4] gives details of their derivations, so we do not know what was different in their models. We were not able to reproduce either result. The latest paper utilizing the square-well model (in the weak coupling limit) is Ref. [10]. Our own result for the square-well model reduces to that of Ref. [10] in the weak limit, and reads

$$
\begin{aligned}
& T_{c}=1.14 \omega_{p} \exp \left[-\frac{1+\lambda_{s}+\lambda_{p}}{\lambda_{p}-\frac{\lambda_{s}\left(1+\lambda_{s}\right)}{1+\lambda_{s}+\lambda_{s} \ln \left(\omega_{s} / \omega_{p}\right)}}\right], \\
& T_{c}=1.14 \omega_{s} \exp \left[-\frac{1+\lambda_{s}+\lambda_{p}}{\frac{\lambda_{p}\left(1+\lambda_{p}\right)}{1+\lambda_{p}-\lambda_{p} \ln \left(\omega_{p} / \omega_{s}\right)}-\lambda_{s}}\right],
\end{aligned}
$$

for $\omega_{s} \geq \omega_{p}$ and $\omega_{s} \leq \omega_{p}$, respectively. Unlike Eq. (4), Eqs. (5) and (6) reduce to the McMillan form upon substitution $\omega_{s} \rightarrow \omega_{C} \gg \omega_{p}, \lambda_{s} \rightarrow \mu$, as it should.

Given the controversy about the square-well model, it is desirable to have a derivation in a controllable approxima- tion, such as the real frequency axis formalism of Ref. [9]. Assuming an Einstein phonon at a frequency $\omega_{p}$ and an "Einstein" paramagnon at $\omega_{s}, 2 \alpha^{2} F(\omega)=\lambda_{p} \omega_{p} \delta(\omega-$ $\left.\omega_{p}\right)-\lambda_{s} \omega_{s} \delta\left(\omega-\omega_{s}\right)$, we obtain the following iterative solution of the Eliashberg equations:

$$
\begin{aligned}
T_{c}= & 1.14 \omega_{p}^{\lambda_{p} /\left(\lambda_{p}-\lambda_{s}\right)} \omega_{s}^{-\lambda_{s} /\left(\lambda_{p}-\lambda_{s}\right)} \\
& \times \exp (K) \exp \left\{-\frac{1+\lambda_{p}+\lambda_{s}}{\lambda_{p}-\lambda_{s}-\mu^{*}\left(1-K \frac{\lambda_{p}-\lambda_{s}}{1+\lambda_{p}+\lambda_{s}}\right)}\right\}, \\
K= & -\frac{1}{2}-\frac{\lambda_{p} \lambda_{s}}{\left(\lambda_{p}-\lambda_{s}\right)^{2}}\left[1+\frac{\omega_{p}^{2}+\omega_{s}^{2}}{\omega_{p}^{2}-\omega_{s}^{2}} \ln \frac{\omega_{s}}{\omega_{p}}\right] .
\end{aligned}
$$

For $\omega_{p} \rightarrow \omega_{s}, K=-1 / 2$, and at $\mu^{*}=0$, Eq. (7) reduces to Eq. (4) with $\nu=\lambda_{p} /\left(\lambda_{p}-\lambda_{s}\right)$.

As usual, the ultimate test for any approximation is numerical calculations. We solved the Eliashberg equations for a variety of model $\alpha^{2} F(\omega)$ including SF and compared them with the proposed analytical formulas. In Fig. 1 we show this comparison for the simplest "onemode" approximation, one phonon and one paramagnon (we have verified that other model spectra lead to similar results). As we can see, while Eq. (7) as well as its simplified version Eq. (4a) describe the numerical results rather well when $\omega_{s}$ and $\omega_{p}$ are comparable, the latter fails at $\omega_{s} \gg \omega_{p}$, and both fail at $\omega_{s} \ll \omega_{p}$. Both effects can be easily understood: Eq. (4) includes $\omega_{s}$ in a negative power in all regimes, thus leading to a total suppression of superconductivity at $\omega_{s} \rightarrow \infty$. In reality, in this limit the negative effect of the SF is renormalized down logarithmically in the same spirit as the Coulomb repulsion.

Equations (4) and (7) diverge at $\omega_{s} \rightarrow 0$. This is due to the fact that the derivations above assume that $\omega_{s}, \omega_{p} \gtrsim$ $\pi T_{c}$. It is possible to treat this regime separately. If $\omega_{s} \ll$ $T_{c}$, the SF act as static magnetic defects, and the standard theory of the magnetic pair breaking [11] can be applied. Thus, at $\omega_{s}=0$ one needs only to keep the term with $n=$ $m$ in $\lambda_{s}\left(\omega_{n}-\omega_{m}\right)$. Then the equations reduce to the standard form [5,11] with the pair-breaking parameter $\gamma \equiv$ $\left(1 / 2 \tau_{P}\right) / \pi T_{c}=\lambda_{s}$. In the weak coupling limit, $T_{c}$ is

$$
T_{c}=T_{c 0} \exp [\psi(1 / 2)-\psi(1 / 2+\gamma)],
$$

where $T_{c 0}=T_{c}\left(\lambda_{s}=0\right)$. One important difference exists between pair breaking by SF with $\omega_{s}=0$ and by magnetic impurities: in the former case, the pair-breaking parameter $\gamma$ now does not depend on $T_{c}$. This has consequences for the isotope effect, as we will see below.

For small but finite $\omega_{s} \ll \pi T_{c}$ summation of $\lambda_{s}(n-m)$ over $n-m$ provides the expression for the pair-breaking rate in Eq. (8): $\gamma=\lambda_{s} \frac{T_{c}}{2 \omega_{s}} \operatorname{coth} \frac{T_{c}}{2 \omega_{s}}$. This result coincides with Eq. (5.8) of Ref. [12] for dynamical pair breaking in anisotropic superconductors if the anisotropy parameter $g$ (as defined in Ref. [12]) is set to -1 . When $\omega_{s}$ increases, $T_{c}$ drops sharply with a complete loss of superconductivity at $\omega_{s}=\omega_{s}^{*}=e^{-C} T_{c 0} / 2 \gamma$ (where $e^{C} \simeq 1.78$ ). However, 


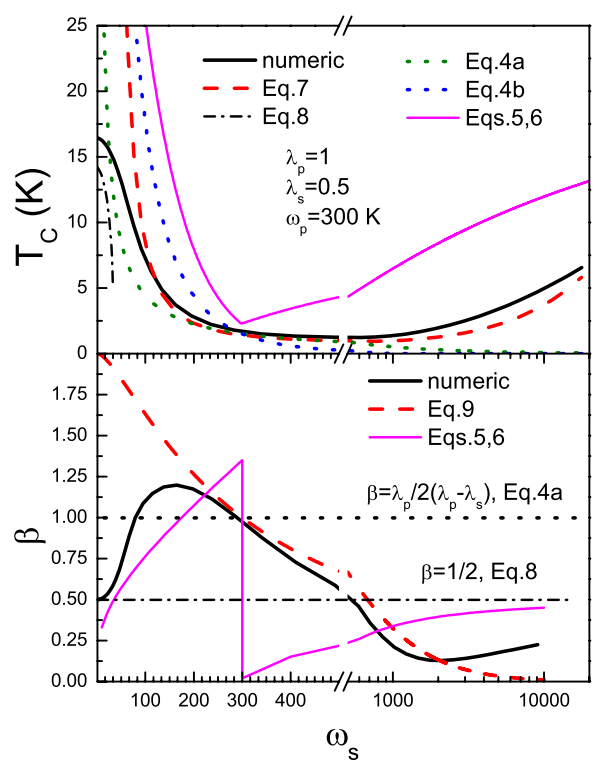

FIG. 1 (color online). Comparison of $T_{c}$ and isotope coefficient with the exact numerical calculations. Note that both Eq. (4b) (the upper broken line) and the square-well model [Eqs. (5) and (6)] disagree qualitatively with the numerical results in the whole range of $\omega_{s}$.

the condition $\omega_{s} \ll \pi T_{c}$ used in the derivation of Eq. (8) is lost well before $\omega_{s}^{*}$ (in fact, at $\omega_{s} \simeq \omega_{s}^{*} / 2$ ).

One can take into account the strong coupling effects in the square-well model, resulting in a renormalization $\gamma \rightarrow$ $\gamma /\left(1+\lambda_{p}\right)=\frac{\lambda_{s}}{1+\lambda_{p}} \frac{T_{c}}{2 \omega_{s}} \operatorname{coth} \frac{T_{c}}{2 \omega_{s}}$. As the comparison with numerical calculations shows (Fig. 1), this approximation underestimates $T_{c}$. However, it illustrates why $T_{c}$ flattens out at a finite value smaller than $T_{c 0}$, when $\omega_{s} \rightarrow 0$, instead of raising as Eq. (7) suggests.

We will now turn to the isotope effect. Looking at Eq. (4), one observes that the isotope coefficient, $\beta=$ $\nu / 2=\lambda_{p} / 2\left(\lambda_{p}-\lambda_{s}\right)>0.5$, is always enhanced compared to its BCS value and is independent of the SF frequencies. Clearly, this should hold approximately in the range of the applicability of this formula, $\omega_{s} \simeq \omega_{p} \gg$ $\pi T_{c}$. Indeed, the more accurate Eq. (7) yields for $\beta$

$$
\begin{aligned}
\beta & =0.5 \frac{\lambda_{p}}{\lambda_{p}-\lambda_{s}}\left[1-\frac{\lambda_{s}}{\lambda_{p}-\lambda_{s}} F\left(\frac{\omega_{s}^{2}}{\omega_{p}^{2}}\right)\right], \\
F(r) & =\left(r^{2}-2 r \ln r-1\right) /(r-1)^{2} .
\end{aligned}
$$

The second term is the correction to Eq. (4). It can be of either sign. [The $F(r)$ monotonically grows from -1 to 1 , and $F(1)=0$.] As discussed, Eq. (4) itself becomes invalid at $\omega_{s}<\pi T_{c}$. As $\omega_{s} \rightarrow 0$, according to Eq. (8), $\beta=0.5$ (note that in the case of magnetic impurities $\beta>0.5$ due to the dependence of $\gamma$ on $T_{c}$ [5]). Therefore, the isotope effect has to have a maximum at some $0<\omega_{s}<\omega_{p}$, and $\beta_{\max }>\lambda_{p} / 2\left(\lambda_{p}-\lambda_{s}\right)[13]$. This is confirmed by numerical calculations, which do show that the maximum isotope effect for given $\lambda_{s}, \lambda_{p}$ is achieved at $\omega_{s} \sim \omega_{p}$ and is close to $\lambda_{p} / 2\left(\lambda_{p}-\lambda_{s}\right)$. Let us emphasize this result: if superconductivity is depressed by spin fluctuations, the total isotope effect increases compared to its BCS value.

We shall now apply this formalism to a superconductor where $T_{c}$ is substantially suppressed by $\mathrm{SF}, \mathrm{MgCNi}_{3}$ $[7,14,15]$. It has attracted substantial interest despite its modest critical temperature, $T_{c} \approx 8 \mathrm{~K}$, but because of its unusual crystal structure and proximity to ferromagnetic instability. The latter was first pointed out by Rosner et al. [7], who believed in such strong coupling with SF that they proposed a $p$-wave superconductivity. Singh and Mazin [14] also concluded that SF play a role in superconductivity of $\mathrm{MgCNi}_{3}$, but, based on their frozen phonon calculation, they found strong coupling with the phonons $\left(\lambda_{p} \gtrsim 1\right)$ due to the bond-bending Ni phonons. They reconciled this large $\lambda_{p}$ with a modest $T_{c}$ within a scenario of $s$-wave phonon-induced superconductivity depressed by SF. Later this scenario was reinvented by Shan et al. [15], who proved the $s$ symmetry by tunneling experiments. This point has been since confirmed by several groups and seems to be well established.

The prediction [14] of the Ni phonon playing the major role in the electron-phonon coupling in $\mathrm{MgNiC}_{3}$ was based on a limited number of calculations at a high-symmetry point in the Brillouin zone, and therefore was more an educated guess than a quantitative argument. A quantitative analysis was provided in Ref. [16] via linear-response calculations of the phonon frequencies and their coupling with electrons for the whole Brillouin zone. They found a gigantic coupling for the $\mathrm{Ni}$ bond-bending modes, and the most strongly coupled modes (the mode considered in Ref. [14] was not among them) actually unstable. In other words, they found double-well-type instabilities involving mostly $\mathrm{Ni}$ atoms, and they have verified that experimentally. They estimated that $\lambda_{p} \approx 0.5$ and $\omega_{\log } \approx 131 \mathrm{~K}$.

Thus, the scenario of Ref. [14] was modified in Ref. [16] in the sense that electron-phonon coupling and superconductivity were coming from highly anharmonic predominantly Ni modes, but not exactly the simple rotations of the $\mathrm{Ni}_{6}$ octahedra considered in Ref. [14]. Strong anharmonicity of these modes makes it impossible to evaluate their coupling with electrons in the linear-response calculations, but it is obviously strong. However, one can estimate the coupling with phonons and paramagnons indirectly from experimental data. Indeed, specific heat renormalization ranges from 2.6 to 3.1 (see Ref. [17] and references therein), implying that the sum $\lambda_{p}+\lambda_{s}$ varies between 1.6 and 2.1. Wälte et al. [17] estimated $\omega_{p} \approx$ $143 \mathrm{~K}$, comparable to the calculation in Ref. [16], $\omega_{s} \approx$ $25 \mathrm{~K}$, and $\lambda_{s} \approx 0.43$. Then, using MMF, $\mu^{*}=0.13$ and $T_{c}=6.8 \mathrm{~K}$, as measured for their samples, they deduced $\lambda_{p}=1.91$.

However, there are several problems with this derivation. First of all, as shown above, the proper formula is Eq. (4). Using it instead of Eq. (2), and keeping all their other parameters, we get a more realistic number, $\lambda_{p}=1.6$ 


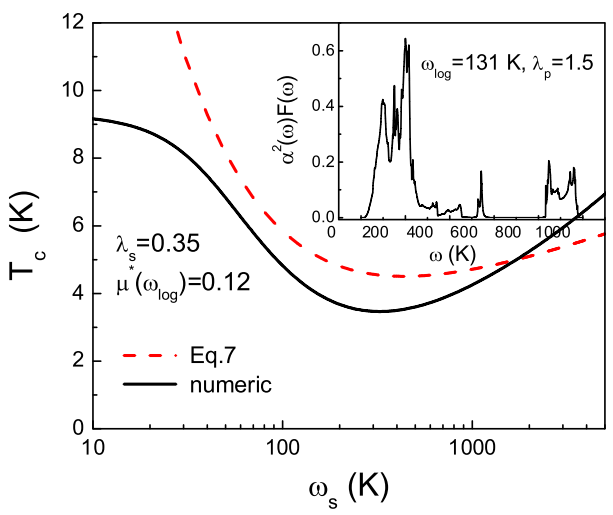

FIG. 2 (color online). $T_{c}$ for the electron-phonon spectral function calculated in [16] for $\mathrm{MgCNi}_{3}$ (inset).

(cf. $\lambda \sim 1.5$ obtained in Ref. [16]). However, the SF model adopted in Ref. [17] cannot be considered as proven. It is based on the disputable assumption that the upturn of the specific heat quotient at low temperature and high magnetic field is due to the paramagnon contribution to specific heat, while there many other explanations of this effect. $\omega_{p} \sim 25 \mathrm{~K}$ seems to be unrealistically soft. Also, low $T_{c}$ and high residual resistance cast doubt on the sample quality in this study.

Here we use a different approach: we adopt the calculated values $\lambda_{p}=1.5$ and $\omega_{p}=131 \mathrm{~K}$, in the harmonic approximation, and total mass renormalization $1+\lambda_{p}+$ $\lambda_{s}=2.85$, so that $\lambda_{s}=0.35$. The results of the numerical solution of the Eliashberg equations with the $\alpha^{2} F(\omega)$ from Ref. [16] and $\mu^{*}=0.12$ are shown in Fig. 2, together with the curve calculated from Eq. (7). This way, we find $\omega_{s} \sim$ $50 \mathrm{~K}$, which we believe is a more realistic number than $25 \mathrm{~K}$. The corresponding total isotope effect is 0.75 .

This may sound in agreement with the recent experiment by Klimczuk and Cava [18], who have measured the isotope effect $\beta_{\mathrm{C}}=0.54$ on carbon only. If the total $\beta=$ 0.75 , this suggests for $\mathrm{Ni}$ a seemingly reasonable $\beta_{\mathrm{Ni}}=$ 0.21 , suggesting that $\mathrm{Ni}$ phonons couple with the electrons twice weaker than $\mathrm{C}$ ones. Unfortunately, the firstprinciples calculations indicate that the $\mathrm{Ni}$ modes couple with electrons at least an order of magnitude stronger than the $\mathrm{C}$ modes (there is hardly any $\mathrm{C}$ character present at the Fermi level). Currently, the only way to reconcile this with the measurements of Ref. [18] is to assume that the observed isotope effect is not a result of the frequency shift of the $\mathrm{C}$ modes, but of some subtle changes in the crystal structure induced by the isotope substitution. Such a possibility is suggested by an earlier study [19], where it was found that (i) $T_{c}$ depends on the lattice parameter at a rate of $\approx 310 \mathrm{~K} / \AA$, which translates an error of $\pm 0.0015 \AA$ in the lattice parameter [18] into an error of $\pm 0.46 \mathrm{~K}$ in $T_{c}$, larger than the isotope shift of $0.3 \mathrm{~K}$, and (ii) that two samples with the same lattice parameter and the same neutron-measured $\mathrm{C}$ content have $T_{c}$ differing by $0.71 \mathrm{~K}$. A possible explanation is that, given the proximity of $\mathrm{MgCNi}_{3}$ to a ferromagnetic instability, crystallographic defects may induce local magnetic moments which work as pair breakers. The concentration of such defects, even for the same $\mathrm{C}$ content, may depend on the sample preparation and, possibly, on isotope substitution. Therefore further studies of the isotope effect on both $\mathrm{C}$ and $\mathrm{Ni}$ are necessary, in particular, combined with accurate measurements of the isotope shift of the phonon modes.

We acknowledge support from the NWO-RFBR Grant No. 047.016.005 and from the NSF DMR Grants No. 0342290 and No. 023188.

[1] N. F. Berk and J. R. Schrieffer, Phys. Rev. Lett. 17, 433 (1966); G. Gladstone, M. A. Jensen, and J. R. Schrieffer, in Superconductivity, edited by R. D. Parks (Marcel Dekker, New York, 1969), Vol. 2, Chap. 13.

[2] H. Rietschel and H. Winter, Phys. Rev. Lett. 43, 1256 (1979).

[3] S. V. Vonsovsky, Yu. A. Izyumov, and E.Z. Kurmaev, Superconductivity of Transition Metals, Their Alloys and Compounds (Springer-Verlag, Berlin, 1982).

[4] P. J. Williams and J. P. Carbotte, Phys. Rev. B 43, 7960 (1991); E. J. Nicol and J. P. Carbotte, Phys. Rev. B 44, 12511 (1991).

[5] P. B. Allen and B. Mitrović, in Solid State Physics, edited by H. Erenreich, F. Zeitz, and D. Turnbull (Academic, New York, 1982), Vol. 37, p. 1.

[6] I. I. Mazin, D. A. Papaconstantopoulos, and M. J. Mehl, Phys. Rev. B 65, 100511(R) (2002).

[7] H. Rosner et al., Phys. Rev. Lett. 88, 027001 (2002).

[8] T. Jarlborg, Physica (Amsterdam) 385C, 513 (2003).

[9] A.E. Karakozov, E. G. Maksimov, and S. A. Mashkov, Sov. Phys. JETP 41, 971 (1976); E. G. Maksimov and D. I. Khomskii, in High-Temperature Superconductivity, edited by V. L. Ginzburg and D. A. Kirzhnits (Consultants Bureau, New York, 1982), Chap. 4.

[10] H. Shimahara, J. Phys. Soc. Jpn. 72, 1851 (2003).

[11] A. A. Abrikosov and L.P. Gor'kov, Sov. Phys. JETP 12, 1243 (1961).

[12] A. J. Millis, S. Sachdev, and C. M. Varma, Phys. Rev. B 37, 4975 (1988).

[13] This is not the only case where electronic interactions can enhance isotope effect; cf., e.g., T. Dahm et al., Phys. Rev. B 61, 6381 (2000).

[14] D. J. Singh and I. I. Mazin, Phys. Rev. B 64, 140507 (2001).

[15] L. Shan et al., Phys. Rev. B 68, 144510 (2003).

[16] A. Y. Ignatov, S. Y. Savrasov, and T. A. Tyson, Phys. Rev. B 68, 220504(R) (2003).

[17] A. Wälte et al., Phys. Rev. B 70, 174503 (2004).

[18] T. Klimczuk and R. J. Cava, Phys. Rev. B 70, 212514 (2004).

[19] T. G. Amos et al., Solid State Commun. 121, 73 (2002). 